\title{
"And Now Close Your Eyes or Lower Your Gaze": Exploring Novice Meditators and Their Attentional Processes During Meditation
}

\author{
Isabella Sprawson ${ }^{1} \cdot$ Jeffrey Wood ${ }^{1} \cdot$ Michail Mantzios ${ }^{1}$ \\ Received: 23 February 2020 / Accepted: 4 May 2020 / Published online: 30 May 2020 \\ (C) The Author(s) 2020
}

\begin{abstract}
Brief mindfulness meditation practices are associated with a wealth of benefits; however, factors that may influence the success of meditation sessions have rarely been explored. The present study explored the effects of the visual environment as a factor of successful meditation. Eye-tracking techniques were employed to objectively measure attention within three attention-deviating conditions with basic meditation instructions, and the potential influence of personality traits as assessed through administering the HEXACO-60-PI, a self-report measure, to participants. Statistically significant results were uncovered regarding decreased fixation durations and increased state mindfulness scores of participants within the blank screen conditions over the two eyesopen conditions. No significant effect was found regarding fixation counts, which decreased within the blank screen condition. The findings regarding reduced state anxiety did not reach significance and there were no significant differences regarding the six personality types between conditions. The present study offers a step towards understanding how brief mindfulness meditation sessions can be optimised.
\end{abstract}

Keywords Mindfulness $\cdot$ Attention $\cdot$ Eye tracking $\cdot$ HEXACO $\cdot$ Visual environment

\section{Introduction}

Research into mindfulness meditation has illustrated a host of enhancements to health and wellbeing, and it is unsurprising that there is increased interest within both clinical and empirical fields regarding the potential benefits. Evoking a mindful state is often triggered through various meditation techniques derived from Buddhist traditions (Bishop et al. 2004), and mindfulness meditation is described as a process of attending to present moment experiences, with an open, accepting and non-judgemental attitude (Kabat-Zinn 2003). Brief mindfulness practices have been shown to immediately and significantly lower stress levels (Mohan et al. 2011), as well as reduce fatigue and anxiety (Zeidan et al. 2010). Some empirical evidence has suggested that long-term psychological difficulties, such as trait anxiety, are better treated by long-term mindfulness-based interventions, but brief interventions have

Michail Mantzios

michael.mantzios@bcu.ac.uk

1 Department of Psychology, Faculty of Business, Law and Social Sciences, Birmingham City University, Room C307, The Curzon Building, 4 Cardigan St., Birmingham B4 7BD, UK shown to significantly reduce short-term state anxiety and increase state mindfulness (Bergen-Cico et al. 2013). Meanwhile, short practices are compiled together to form larger programs, and are significant in achieving the optimal outcome for people who take part in such programs. Therefore, brief mindfulness practices have important implications for short-term improvements in health and wellbeing, as well as long-term programs, and should be thoroughly explored to improve their overall success. In this research, we explore two factors within mindfulness meditation that may influence the effectiveness of the practice itself, namely, personality and visual distraction.

There are several influences that appear to affect the effectiveness of mindfulness-based interventions, and research is beginning to investigate the role of personality regarding both state and trait mindfulness (de Vibe et al. 2015). For example, openness to experience, agreeableness and conscientiousness have been positively associated with mindfulness, whereas neuroticism (i.e., the personality trait most associated with anxiety, selfconsciousness and insecurity (Lee et al. 2006) has consistently shown to be negatively related to mindfulness (Giluk 2009). These personality traits are often defined by the five-factor personality model (see McCrae and Costa 1987). The five-factor model of personality, or otherwise known as the Big Five, 
includes the following factors: (I) extraversion, (II) agreeableness, (III) conscientiousness, (IV) emotional stability (versus neuroticism) and (V) openness to experience. Highly neurotic individuals are increasingly distracted during attentional tasks, through entertaining personal thoughts and allowing the mind to wander (Robison, Gath \& Unsworth, 2016), potentially leading to little success during mindfulness meditation.

Neuroticism correlating strongly to mindfulness is unsurprising, although the exact relationship is unclear. To date, research has not determined whether being mindful decreases neurotic susceptibility, or whether neuroticism interferes with present-moment awareness (Thompson and Waltz 2007) and decreases the effectiveness of mindfulness-based interventions. Existing research has illustrated that increased anxiety may be related to lower cognitive performance (Eysenck et al. 2007) and through experience-sampling studies whereby participants are randomly asked to complete questionnaires about their immediate experiences, findings have demonstrated how anxious individuals tend to report increased inattentiveness (Kane et al. 2007). Therefore, personality may be a factor that can influence the success of meditation, and neuroticism may stretch even to misinterpreting or adding unnecessary meaning to the guidance that is usually provided during meditation sessions.

During guided meditation practice, individuals are often instructed to both lower and soften their gaze, or close their eyes entirely, and research has not yet explored whether there is a difference between choosing one over the other. Indeed, one particularly relevant factor that may influence the effectiveness of mindfulness-based interventions includes the surrounding visual environment, and the ability to sustain focused attention on the present moment. Regrettably, simply instructing individuals to become aware of and accept taskirrelevant stimuli may not be sufficient to prevent visual distractors to attentional processes, resulting in poorer meditation experiences. Experimental research manipulating taskirrelevant distractions within the visual environment has demonstrated interruptive effects on performance, even in situations where participants are explicitly told to overcome irrelevant material through non-judgement (Forster and Lavie 2008). During guided mindfulness breathing meditation, when individuals are instructed to lower their gaze, they are guided to focus on their breath and the changing sensations of the present moment (Wong et al. 2015), thereby ignoring the distractions of the visual environment. Both seminal and recent researches have demonstrated how gaze aversion and eye closure have shown to improve memory recall (Glenberg et al. 1998; Perfect et al. 2011). From the limited existing research, simply lowering the gaze leaves the meditating individual exposed to the distractors within the visual environment, and may hinder the experience through taking focus away from the breath and leading to attention to be additionally distracted from the meditation than if the eyes are closed.
Emerging studies are beginning to employ the use of eye tracking as an objective measure of distraction and attention, based on the assumption that the eye movement reflects what processes are occurring within the mind. Significantly, the scene that the individual is watching is an element of importance, especially when considering meditation and the self-regulation of attention, and proposes a question of semantics in meditation guidance and instructions. Eye movements appear to differ during instances of distraction, particularly when compared with on-task eye movements (Foulsham et al. 2013). Distraction is particularly associated with more frequent eye blinks (Smilek et al. 2010) and divergent or erratic eye movements (Foulsham et al. 2013; Reichle et al. 2010). Although many eye movement studies in distracted attention are in relation to mindless reading, one particular study into the saccadic movements, both catch-up and anticipatory saccades and smooth pursuit eye movements between experienced meditators and non-meditators, uncovered potential attentional differences. The reduced catch-up saccades amongst experienced meditators suggested improved control of eye movements and related to improved attentional control (Kumari et al. 2017) that is a commonly reported foundational element of mindfulness meditation (see Schreiner and Malcolm 2008).

Despite increasing research into mindless reading using ocular movement as an objective measure of distracted attention, little is known regarding the link between eye and mind during meditation. It may be presumed that the eyes play only a small functioning role in meditation, particularly as meditation is often performed with the eyes closed. Feeling comfortable to keep the eyes closed may however not be the case for people who score higher in neuroticism; indeed, some people prefer to remain in control by keeping their eyes open. Only one study has recently investigated ocular movements relating to mind wandering and meditation, with findings demonstrating higher levels of ocularmetric measures during episodes of mind wandering (Matiz et al. 2019). Matiz et al. (2019) further suggested that through employing the use of eye-tracking technology during mindfulness meditation, results can provide a more objective insight into mindful mediation. Their research demonstrated that mind-wandering instances are associated with greater levels of ocular activity through comparing eye movements between participants within a focused-breath meditation session or an instructed mind-wandering task. Thus, the research proposes that high levels of ocular movement during meditation practices are related to instances of mind wandering, though to date there is no evidence directly investigating mindfulness meditation that is performed under differing conditions (e.g. eyes-open meditation versus eyesclosed meditation). 


\section{The Current Study}

The present study has a number of aims to address the lack of attention into increased distraction during mindfulness meditation. To date, research is yet to investigate which personality types are particularly vulnerable to being distracted during mindfulness meditation. From existing literature, it is expected that participants scoring highly on the emotionality scale, which is particularly related to increased anxiety and fear, will experience increased instances of distraction and perhaps a less successful meditation session. Secondly, the present study aims to further investigate whether mindfulness meditation is more successful when there are no visual distractions. Given both the existing literature into mind wandering and the literature on the effect of distractors in the visual field have employed the use of eye tracking, eye movements will be monitored as an objective measure of attention during mindful meditation.

We predict that participants given an image depicting a lowered gaze perspective might be increasingly challenged by visual distraction and increased instances of mind wandering. Results are expected to produce reduced anxiety and increased mindfulness scores within the closed-eye perspective condition, and higher anxiety scores with lower mindfulness scores amongst the patterned screen and lowered gaze perspective conditions. Eye movements are also expected to show increased fixation times outside a fixation point when participants are given an image depicting the lowered gaze. In comparison, participants given an image reflecting eye closure will have no visual distractors and will show less fixations away from the fixation point. Research in other fields has shown eye tracking provides a good measure of attention. They show that when distractors are present, attention is drawn away from the task at hand. For example, research on distractors when driving (Dukic et al. 2013) showed that number of fixations and fixation duration increased when certain objects were present in a driver's visual field. Addressing the lack of research into visual distraction during meditation specifically, depending on eye closure, will benefit future instructors of mindfulness meditation and provide a better insight into how meditators should be instructed. Through assessing personality dimensions and the potential differences regarding their experiences, the present study may offer some clarification on whether mindfulness meditation is more successful when specific personality types are evident.

\section{Method}

\section{Participants}

Forty-eight participants were recruited to take part in this study. The ages of participants ranged from 18 to $33(M=$
21 years, $S D=2.7)$. Fourteen participants had prior experience with meditation, including mindfulness, self-compassion, yoga and breath-focused meditation, similarly distributed across experimental groups $(4=$ eyes closed, $4=$ patterned, $6=$ lowered gaze). From the 14 participants with prior meditation experience, only 1 participant practiced some form of meditation daily in the lowered gaze condition. Seven participants also reported a diagnosis of a mental health disorder, including anxiety, depression and PTSD $(4=$ eyes closed, $2=$ patterned, $1=$ lowered gaze). Analyses presented in this manuscript explored the potential differences in findings by excluding participants with prior experience, or with a mental health diagnosis, but results did not differ. Participants were recruited both through opportunistic sampling and through a Research Participation Scheme (RPS), whereby participants who were recruited through this scheme were awarded credits in return for their participation.

\section{Materials}

Demographics Questionnaire A demographics questionnaire was allocated to all participants. This questionnaire collected basic background information, including age, gender and ethnicity of participants. Participants were also asked to detail any diagnosis of a mental health disorder and medication, and whether they had any previous or current experience with meditation. This information was collected in order to ensure the participation sample was representative, and to identify potential reasons behind any outliers in the data.

\section{HEXACO-60 Personality Inventory-Revised (HEXACO-60-PI-R)} The HEXACO-60-PI-R (Ashton and Lee 2009) was allocated to participants as the first questionnaire to complete. The purpose of this self-report questionnaire was to assess the six major personality dimensions within participants. The personality dimensions measured by this questionnaire are as follows: honesty/humility (H), emotionality (E), extraversion (X), agreeableness versus anger (A), conscientiousness (C) and openness to experience $(\mathrm{O})$. The inventory consists of 60 statements, including "I often push myself very hard when trying to achieve a goal" and "I prefer jobs that involve active social interaction to those that involve working alone". Responses were recorded using a 1 to 5-point Likert Scale, ranging from 1 (strongly disagree) to 5 (strongly agree). Despite being a shortened version of the HEXACO-PI-R, which consists of 200 items, the HEXACO-60-PI-R has demonstrated consistent internal reliabilities with Cronbach's Alpha scores across each personality dimension exceeding .70 .

State Mindfulness Scale The State Mindfulness Scale (SMS) was allocated to participants before and after the brief meditation session and intended to measure the state mindfulness. The SMS is a 21-item self-report measure and includes 
statements such as "I tried to pay attention to pleasant and unpleasant sensations" and "I found some of my experiences interesting". The items intend to measure both the objects of mindful attention, or rather what physical and mental sensations are being attended to as well as how an individual is attending to the present moment (Tanay and Bernstein 2013). Responses were recorded using a 5-point Likert scale: 1 (strongly disagree) to 5 (strongly agree). The SMS has consistently demonstrated strong internal reliabilities $(\alpha=$ $.90-.95$ ), for both mental and physical factors, as well as the scale as a whole. Strong convergent, divergent and incremental validities have also been established (Hadash et al. 2016). The SMS was deemed the most appropriate scale to utilise for this experiment, as alternative state mindfulness scales could not suitably measure state mindfulness during meditation (Tanay and Bernstein 2013).

Shortened State Anxiety Scale A 6-item short form of the Spielberger State - Trait Anxiety Inventory (STAI; Spielberger et al. 1970) had been administered to participants. This scale includes statements, such as "I feel calm" and "I feel tense" (Marteau and Bekker 1992). Similar to the SMS, a 5-point Likert scale was utilised to record responses. The short-form scale was developed to measure state anxiety alone, for instances in which the full form is deemed inappropriate to use. The short form allowed this experiment to attain specific measurement of state anxiety, reduce participant fatigue and has demonstrated strong internal consistency reliability and validity when correlated with the full-form 20 -item State Anxiety Scale (Tluczek et al. 2009).

Mindfulness Audio File Participants were provided with a short 20-min audio file (Mantzios 2018), which included directions for a guided mindful breathing practice. The only difference that occurred in this meditation was that the focus in breath was aligned with a further instruction by the researcher to maintain the visual attention to the square.

Images/Stimuli Participants were asked to hold their gaze on one of the potential three images presented to them. The first condition consisted of a basic black screen that intended to imitate an eyes-closed perspective. The second and third images, taken with a $12 \mathrm{MP}$ camera, were depicting a lowered gaze. One image simply consisted of flooring, with no other distractors within the image. The second image depicted a lowered gaze whereby the floor was included, as well as other distractors (i.e. chairs, legs and shoes). Each image had a small white square created in MSPaint, positioned in precisely the same location, roughly in the upper middle of each image (see Fig. 1). The areas of interest for each image included all of the rest of the screen excluding the white square and a very narrow window around this to account for eye drift.
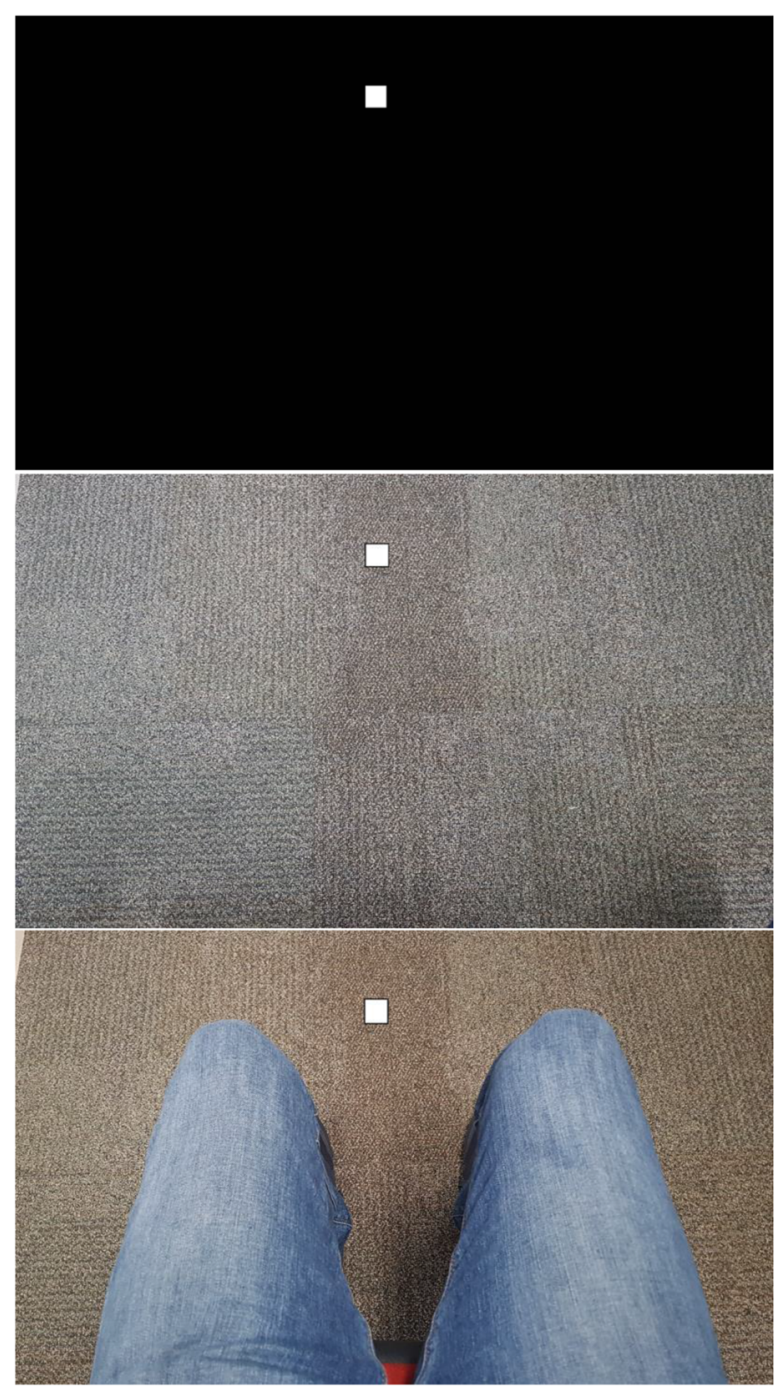

(b)

Fig. 1 Images displayed for different conditions (a eyes closed, b patterned, c lowered gaze)

Equipment Eye tracking was performed using the Tobii Pro X3-120 eye tracker (See https://www.tobiipro.com/productlisting/tobii-pro-x3-120/), and this equipment was mounted onto a Lenovo ThinVision Liquid Crystal Display 22" monitor, with a resolution of $1920 \times 1080$ pixels. The eye tracker captured gaze at a sampling rate of $120 \mathrm{~Hz}$. The monitor was positioned comfortably $60 \mathrm{~cm}$ away from participants' eyes (Duchowski 2003). The area of interest was the remainder of the image that excluded the white square participants were instructed to rest their eyes on. The eyetracking equipment only captured eye movement around the screen. All questionnaires and scales participants were provided with were completed online using Qualtrics software.

\section{Procedure}

A short questionnaire was given to participants in order to collect basic demographic information, including age, gender 
and ethnicity. The HEXACO-60-PI-R was the first scale allocated to participants, which was completed online $24 \mathrm{~h}$ before their scheduled experimental timeslot. When participants arrived to complete their participation, they were randomly allocated to one of the three conditions which determined the stimuli they were presented with during the meditation session. The images included a black screen, patterned screen or an image depicting a lowered gaze. Before the short meditation session began, participants were asked to provide responses to both the State Mindfulness Scale and the shortform State Anxiety Scale. Once completed, participants could begin the audio file relating to mindfulness meditation and breathing. Participants were instructed to sit in an upright, yet relaxed, position and soften their gaze while attending to the image presented to them for the entirety of the session. Within the first condition, participants were allocated to a black screen to look at during their meditation, which intended to mimic an eyes-closed perspective, whereas the second and third conditions were allocated a patterned image and an image depicting a lowered gaze, respectively. Eye movements of participants were recorded throughout the meditation session. After participants had completed the meditation audio file, they were asked to complete the SMS and short-form State Anxiety Scale once more.

\section{Analysis}

The results will be analysed predominantly using one-way ANOVAs. Personality will be assessed to determine whether specific personality types were more evident within each condition. One-way ANOVAs will also assess whether fixation durations were different across the three conditions. Further one-way ANOVAs will be carried out in order to investigate whether the total fixation counts were different across the three conditions, and whether the average fixation durations across the three conditions differ. Two 3 (condition: closed, lower, pattern) $\times 2$ (time: pre, post) mixed design ANOVAs will be conducted to analyse the state mindfulness and state anxiety scores.

\section{Results}

The analyses were completed by comparing the areas of interest, which included the image participants were presented with the exception of the fixation square and a small area around this white fixation square. Three processing measures were analysed. The total fixation durations refer to the sum of fixation durations in the area of interest, measured in seconds. The average fixation duration measured the average amount of time participants' eyes spent in the area of interest per each fixation and finally, the fixation counts measured the sum total of instances participants' eyes fixated away from the fixation cross.
One-way ANOVAs were performed in order to determine whether specific personality types, as assessed by the HEXACO-60-PI, were more evident in each condition. Homogeneity of variances was not found to be violated for the present analysis. The ANOVA revealed no significant differences between any of the six personality types: honesty/humility $(p=.849)$, emotionality $(p=.981)$, anxiety $(p=.643)$, extraversion $(p=.434)$, openness to experience $(p$ $=.842)$ and conscientiousness $(p=.766)$.

A one-way ANOVA was carried out to determine whether the total fixation durations, measured in seconds, were different across the three conditions that participants were assigned to: closed-eyes perspective $(n=16)$, patterned screen $(n=16)$ and lowered gaze perspective $(n=16)$. The fixation durations recorded how long each participant's eyes were fixated on the area of interest. The means in seconds across the three groups indicate that fixation durations were shorter in the eyes-closed condition $(M=33.60, S D=96.06)$, longer in the lowered gaze perspective $(M=164.12, S D=159.59)$ and longest within the patterned screen condition $(M=187.19, S D=166.54)$ (see Fig. 2). Levene's test for homogeneity of variances was violated for the present analysis, $F(2,45)=6.26, p=.004$. A Welch ANOVA found the differences between conditions and fixation duration were statistically significant, $F(2,27.928)=$ $7.04, p=.003$. Post hoc comparisons were carried out using the Tukey HSD test, and uncovered a statistically significant difference between the eyes-closed condition and the patterned screen condition $(p=.012)$ and a statistically significant difference between the eyes-closed condition and the lowered gaze perspective condition $(p=.036)$. No statistically significant difference was found between the patterned screen condition and the lowered gaze perspective conditions $(p=.894)$.

A second one-way ANOVA was also carried out to investigate whether the total fixation counts were different across the three conditions. The fixation counts measured how many

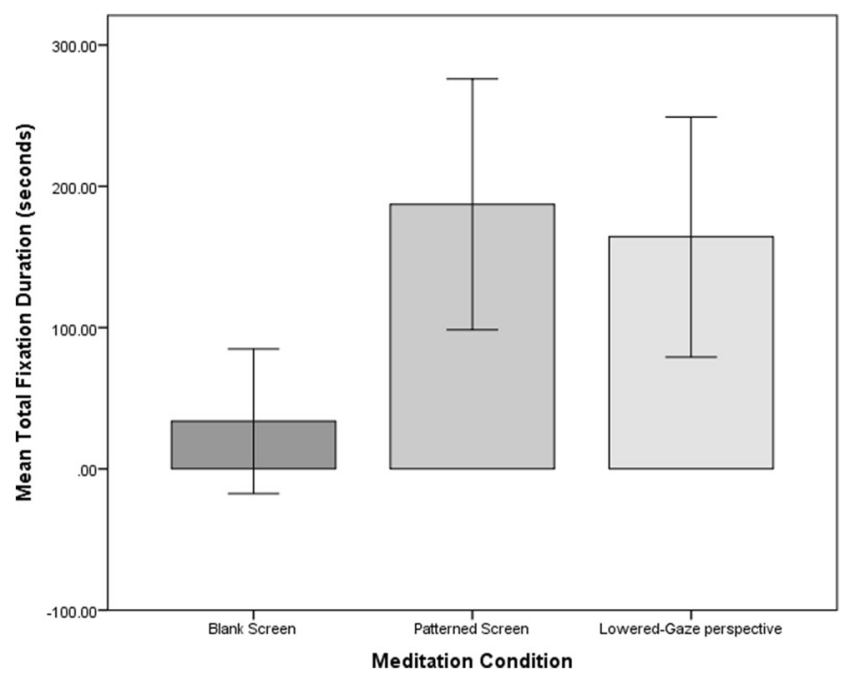

Fig. 2 Means and 95\% confidence intervals for the total fixation durations by condition 
times each participant's eyes diverted into the area of interest. Levene's test for this analysis was not found to be violated, $F(2,45)=1.76, p=.183$. The means across the three conditions indicate similar patterns as in the above analysis, with the eyes-closed perspective condition reporting a lower number of fixation counts $(M=195.43, S D=552.56)$, and increased fixation counts from the lowered gaze perspective $(M=$ $620.81, S D=520.48)$ and the patterned screen $(M=627.31$, $S D=629.57$ ), respectively (see Fig. 3). The analysis, however, found a marginally significant difference between conditions, $F(2,45)=3.02, p=.059$. Post hoc tests (Tukey HSD) were carried out and uncovered no significant difference between the eyes-closed and lowered gaze perspective ( $p=$ .099), and no significant differences between the eyes-closed condition and the patterned screen $(p=.092)$ or the lowered gaze perspective and patterned screen $(p>.999)$.

One final ANOVA was carried out to investigate the average fixation durations across the three conditions. The mean scores indicate lower average fixation duration scores within the eyes-closed condition $(M=152.21, S D=56.11)$, and increased average fixation duration scores within the lowered gaze perspective and patterned screen conditions $(M=245.17$ $S D=87.96$ and $M=351.34, S D=324.89$, respectively) (see Fig. 4). Levene's test for homogeneity of variances was found to be violated $F(2,45)=10.13, p<.001$. The Welch ANOVA found a statistically significant difference between the conditions, $F(2,25.558)=8.34, p=.002$. Using the Tukey HSD post hoc test, significant differences were found between the eyes-closed condition and the patterned screen condition ( $p=$ .017), yet no significant difference was found between the eyes-closed and lowered gaze perspective $(p=.384)$ or between the lowered gaze perspective and the patterned screen conditions $(p=.289)$.

Two 3 (condition: closed, lower, pattern) $\times 2$ (time: pre, post) mixed design ANOVAs were carried out on state mindfulness

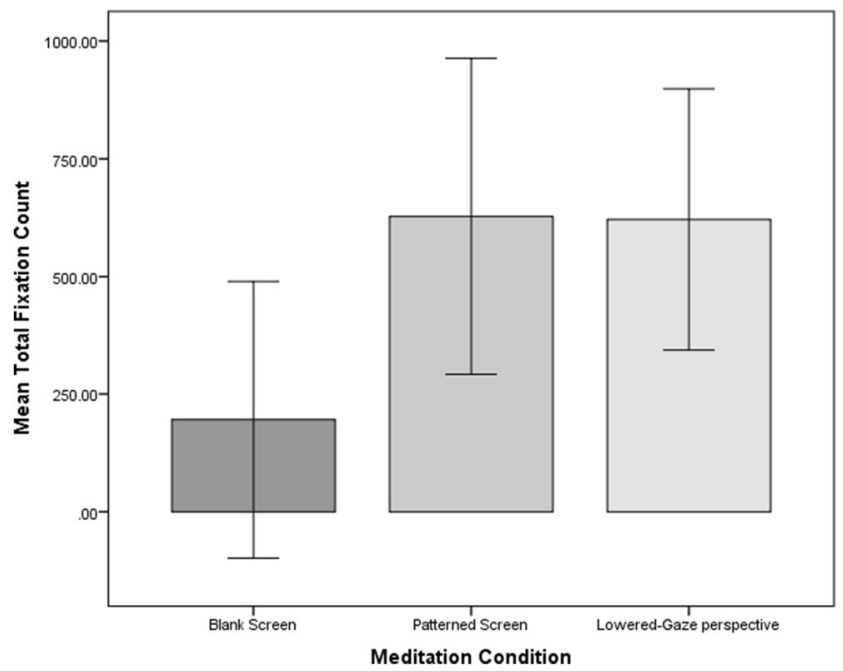

Fig. 3 Means and 95\% confidence intervals for the total fixation count by condition



Fig. 4 Means and 95\% confidence intervals for the average fixation duration by condition

and state anxiety. For state mindfulness, there was a significant interaction between conditions and time $F(2,45)=9.135, p=<$ .001 , significant main effect of time $F(1,45)=19.552, p<.001$, but no significant main effect across conditions $F(2,45)=5.120$, $p=.010$. For state anxiety, there was no significant interaction between conditions and time $F(2,45)=2.585, p=.087$, there was a significant main effect of time $F(1,45)=13.250, p=.001$, but no significant main effect across conditions $F(2,45)=.707, p$ $=.498($ see Table 1$)$.

Follow-up analyses entailed paired sample $t$ tests for each condition to observe changes over time independently for mindfulness and anxiety (see Table 1). Two one-way ANOVAs were also conducted to explore the differences between pre- and post-measurements for mindfulness. For mindfulness, pre- and post-measurements significantly differed, $F(1,45)=6.33, p=.004$ and $F(1,45)=5.68, p=.006$, respectively. Post hoc Tukey HSD indicated that there were significant differences at pre-measurements between pattern and closed-eyes conditions $(\Delta M=20.13, S E=6.18, p=$ $.006, C I-L=5.14, C I-U=35.11)$, as well as pattern and lowered gaze conditions $(\Delta M=17.75, S E=6.18, p=.017$, $C I-L=2.77, C I-U=32.73)$. For post-measurements, the lowered gaze condition differed significantly from the eyesclosed condition $(\Delta M=16.88, S E=5.49, p=.010, C I-L=$ $30.17, C I-U=3.58)$, and the pattern condition $(\Delta M=15.00$, $S E=5.49, p=.024, C I-L=28.30, C I-U=1.70)($ see Table 2). Due to the differences in pre-measurements, an attempt to explore the differences between pre- and post-measurements led to an analysis where the baseline differences would not interfere with the subjective interactive difference between conditions. We therefore calculated the differences between scores (mean pre-mindfulness - mean post-mindfulness $=$ mean mindfulness difference) and explored the mean differences between groups in two one-way ANOVAs. Results indicated that there were significant differences between groups, 
Table 1 Pre- and post-measurements of state mindfulness and state anxiety scores for each condition with paired sample $t$ tests

\begin{tabular}{|c|c|c|c|c|c|c|c|c|}
\hline & Condition & Pre $M(S D)$ & Post $M(S D)$ & $\Delta M$ & $t$ & $p$ & $C I-L$ & $C I-U$ \\
\hline \multirow[t]{3}{*}{ Mindfulness } & Closed-eyes & $64.81(23.04)$ & $88.69(15.47)$ & -23.88 & -5.31 & $<.001$ & -33.45 & -14.30 \\
\hline & Lower & $84.94(11.31)$ & $86.81(14.36)$ & -1.88 & -.50 & .62 & -9.81 & 6.05 \\
\hline & Pattern & $67.19(16.08)$ & $71.81(16.64)$ & -4.63 & -1.28 & .22 & -12.35 & 3.10 \\
\hline \multirow[t]{3}{*}{ Anxiety } & Closed-eyes & $17.88(4.69)$ & $21.06(2.89)$ & -3.19 & -3.08 & .008 & -5.39 & -.98 \\
\hline & Lower & $18.38(4.65)$ & $18.69(5.46)$ & -.31 & -.40 & .69 & -1.97 & 1.34 \\
\hline & Pattern & $16.69(3.57)$ & $18.94(4.37)$ & -2.25 & -2.48 & .03 & -4.18 & -.32 \\
\hline
\end{tabular}

$M$ and $S D$ are used to represent mean and standard deviation, respectively. $\Delta M$, mean difference; $C I-L$, lower confidence interval; $C I-U$, upper confidence interval

where the eyes-closed condition displayed a significantly greater difference when compared with the other two groups, while the rest of comparisons were non-significantly different (see Table 2 for ANOVAs and post hocs).

\section{General Discussion}

The present study had several hypotheses. Firstly, it was predicted that participants who were given an image depicting a lowered gaze perspective would be challenged by the visual distractions within the image resulting in a less successful meditation session. It was also predicted that participants within this condition would demonstrate increased eye movements. Regarding personality, it was hypothesised that participants who scored highly on emotionality may experience increased instances of mind wandering.

Several results from the analyses support the hypothesis of the present study. Firstly, the lengths of fixation durations within the area of interest were measured, which demonstrate how long participants spent exploring the image, rather than focusing on the set white square for their meditation session. The significant results regarding the fixation duration illustrate that participants within the eyes closed spent less time exploring the screen during their meditation session, in line with the initial hypothesis. Fixation counts were also collected, which indicated how frequently participants' eyes were fixated outside of the white square. While the results for the fixation counts were only marginally significant across conditions, the results are consistent with the findings of the fixation duration measures with the least number of fixations away from the fixation point in the closed-eyes condition. The results also indicated that the average fixation duration interfered with the feedback loop or with the self-regulation of attention, where people in both eyes-open conditions spend more time outside the attentional focus as instructed prior and during the meditation. Furthermore, results regarding the closed-eyes condition and the effectiveness of the meditation session were as expected. State mindfulness scores increased within the eyes-closed perspective condition, and these findings were statistically significant when compared with the two other conditions.

The successful meditation that occurred within the eyesclosed condition may be explained in several ways. Firstly, through previous research, the visual environment has demonstrated distracting effects on performance of participants, even when instructed to overcome all irrelevant material through non-judgemental acceptance (Forster and Lavie 2008). These findings can be applied to the present study, as participants within the patterned screen and lowered gaze perspective not only demonstrated lower state mindfulness and
Table 2 Analyses of variance and Tukey post hoc on mean differences (between pre- and post-measurements) by conditions

\begin{tabular}{|c|c|c|c|c|c|c|}
\hline & Condition A & Condition B & $\begin{array}{l}\Delta M \\
\text { Conditions } \mathrm{A} \text { and } \mathrm{B}\end{array}$ & $\begin{array}{l}p \\
\text { Tukey HSD }\end{array}$ & $F$ & $p$ \\
\hline \multirow[t]{4}{*}{ Mindfulness } & Closed-eyes & Lower & 19.25 & .004 & & \\
\hline & Closed-eyes & Pattern & 22.00 & .001 & & \\
\hline & Pattern & Lower & -2.75 & .88 & & \\
\hline & & & & & 9.14 & $<.001$ \\
\hline \multirow[t]{4}{*}{ Anxiety } & Closed-eyes & Lower & .94 & .75 & & \\
\hline & Closed-eyes & Pattern & 2.88 & .08 & & \\
\hline & Pattern & Lower & -1.94 & .30 & & \\
\hline & & & & & 2.59 & .09 \\
\hline
\end{tabular}


state anxiety scores but they also displayed an increased amount of fixation counts and their fixation durations were longer for the patterned screen condition relative to the eyesclosed condition. Therefore, results suggest that the participants in these two conditions were exposed to several visual distractions that may have taken their focus away from their meditation and were less able to bring their focus and attention back to the present moment.

Furthermore, while the initial hypothesis predicted that the lowered gaze perspective would present participants with the most challenging meditation environment, it was the patterned screen that appeared to have the largest adverse effect on the meditation session and may have increased participants' instances of distraction. Participants within the closed-eyes perspective were instructed to rest their gaze on a plain black screen with a single white square to focus on. Within this condition, there was a clear lack of visual distraction that may account for the success. The present study supports previous literature that investigates the closure of the eyes on performance (Glenberg et al. 1998; Perfect et al. 2008), as meditation was most successful within the condition that mimicked a closed-eye perspective. As these results are consistent with existing research, the benefits of eye closure on concentration and performance are thus reinforced, as access to the distractions of the visual environment is eliminated (Perfect et al. 2011).

There was no significant difference in anxiety scores across the conditions. The non-significant results regarding state anxiety were somewhat surprising. As past research has demonstrated clear reductions in anxiety in both experimental (Zeidan et al. 2010) and clinical environments (Kabat-Zinn 1990), it was expected that anxiety scores would be notably reduced within the eyes-closed condition. Nevertheless, it may be the case that participants did not feel particularly anxious prior to their meditation session and it is also particularly important to note that many studies investigating the effectiveness of mindfulness meditation regarding anxiety often use clinical samples consisting of participants diagnosed with known anxiety disorders, or introduce an anxiety-induction element at the beginning of their experiment. Lastly, the results could be explained from the low number of participants in each condition, which may be satisfactory for eye-tracking experiments, but inadequate for behavioural research. Future research could build on the existing experimental paradigm and explore anxiety induction, and/or trait anxiety, and stress as potential indicators of individual differences.

Closely aligned to individual differences is the significant lack of investigation into the role of personality regarding the effectiveness of mindfulness meditation, which was explored through the emerging HEXACO model of personality (see Lee and Ashton 2005). The results regarding personality differences within each condition did not hold any significance. Previous literature has indicated that neurotic personality types are increasingly vulnerable to distracted attention (Robison et al. 2017), and may have difficulty reaping the benefits of mindfulness meditation (Norris et al. 2018). It was expected that participants who scored highly on emotionality may have been increasingly vulnerable to the distraction of the visual environment, and may have experienced a less successful meditation session. It was also expected that openness to experience may have influenced a more successful meditation session. This research should be followed up by increasing the number of participants, and further research selectively exploring people who score high and low in neuroticism or emotionality may inform the potential of creating more inclusive, but also effective practices.

As previously mentioned, one limitation of this research was the small sample size and limited demographic sample, and findings should be approached and interpreted with caution in regard to personality. The present study also investigated the role of personality, but assessing and controlling for trait mindfulness may uncover whether difficulty in engaging with brief mindfulness meditation session is exclusively the case for participants who score low in trait mindfulness. Another interesting path is the auditory distraction that may interfere with the practice, and could potentially be another level of investigation. The idea that "you can practice anywhere" may simply be another element that needs clarity, and may add another barrier to people who are beginners in meditative practices. Also, with practice and experience, the additional distractors that exist within the environment are an additional level of difficulty, but do not obstruct the effectiveness of the practice. While clearly a simplification, an analogy would be of an experienced driver who will struggle less in the midst of traffic than a beginner, but enabling the beginner with less distractors and demands is important to keep them behind the wheel and trying to master the skill of being attentive of the present moment.

\section{Conclusion}

As previous research has not investigated the effect of the visual environment and eye closure on meditation performance, the present study offers a step towards understanding whether mindfulness instructors should direct their clients to close their eyes entirely or simply lower their gaze. While there were no clear personality types that interfered with meditation success, perhaps follow-up studies with larger sample sizes can successfully reveal the role of personality, separately and in combination to eye tracking. The results of the present study offer a significant step forward towards understanding why meditation may be most effective with the eyes closed rather than a lowered gaze, and how the visual environment can influence novice meditators. 


\section{Compliance with Ethical Standards}

The study was approved by the Ethical Review Board of the University, and was in accordance with the ethical standards of the institutional and/ or national research committee, and with the 1964 Helsinki Declaration and its later amendments. This article does not contain any studies with animals.

Conflict of Interest The authors declare that they have no conflict of interest.

Informed Consent Informed consent was obtained from all individual participants included in the study.

Open Access This article is licensed under a Creative Commons Attribution 4.0 International License, which permits use, sharing, adaptation, distribution and reproduction in any medium or format, as long as you give appropriate credit to the original author(s) and the source, provide a link to the Creative Commons licence, and indicate if changes were made. The images or other third party material in this article are included in the article's Creative Commons licence, unless indicated otherwise in a credit line to the material. If material is not included in the article's Creative Commons licence and your intended use is not permitted by statutory regulation or exceeds the permitted use, you will need to obtain permission directly from the copyright holder. To view a copy of this licence, visit http://creativecommons.org/licenses/by/4.0/.

\section{References}

Ashton, M. C., \& Lee, K. (2009). The HEXACO-60: a short measure of the major dimensions of personality. Journal of Personality Assessment, 91(4), 340-345. https://doi.org/10.1080/ 00223890902935878.

Bergen-Cico, D., Possemato, K., \& Cheon, S. (2013). Examining the efficacy of a brief mindfulness-based stress reduction (Brief MBSR) program on psychological health. Journal of American College Health, 61(6), 348-360. https://doi.org/10.1080/ 07448481.2013.813853.

Bishop, S. R., Lau, M., Shapiro, S., Carlson, L., Anderson, N. D., Carmody, J., et al. (2004). Mindfulness: a proposed operational definition. Clinical psychology: Science and Practice, 11(3), 230241. https://doi.org/10.1093/clipsy/bph077.

de Vibe, M., Solhaug, I., Tyssen, R., Friborg, O., Rosenvinge, J. H., Sørlie, T., et al. (2015). Does personality moderate the effects of mindfulness training for medical and psychology students? Mindfulness, 6(2), 281289. https://doi.org/10.1007/s12671-013-0258-y.

Duchowski, A. T. (2003). Eye tracking methodology: theory and practice. New York: Springer.

Dukic, T., Ahlstrom, C., Patten, C., Kettwich, C., \& Kircher, K. (2013). Effects of electronic billboards on driver distraction. Traffic injury prevention, 14(5), 469-476. https://doi.org/10.1080/15389588. 2012.731546.

Eysenck, M. W., Derakshan, N., Santos, R., \& Calvo, M. G. (2007). Anxiety and cognitive performance: attentional control theory. Emotion, 7(2), 336-353. https://doi.org/10.1037/1528-3542.7.2.336.

Forster, S., \& Lavie, N. (2008). Attentional capture by entirely irrelevant distractors. Visual Cognition, 16(2-3), 200-214. https://doi.org/10. 1080/13506280701465049.

Foulsham, T., Farley, J., \& Kingstone, A. (2013). Mind wandering in sentence reading: decoupling the link between mind and eye. Canadian Journal of Experimental Psychology/Revue Canadienne de Psychologie Expérimentale, 67(1), 51-59. https://doi.org/10. 1037/a0030217.

Giluk, T. L. (2009). Mindfulness, Big Five personality, and affect: a meta-analysis. Personality and Individual Differences, 47(8), 805811. https://doi.org/10.1016/j.paid.2009.06.026.

Glenberg, A. M., Schroeder, J. L., \& Robertson, D. A. (1998). Averting the gaze disengages the environment and facilitates remembering. Memory \& Cognition, 26(4), 651-658. https://doi.org/10.3758/ BF03211385.

Hadash, Y., Segev, N., Tanay, G., Goldstein, P., \& Bernstein, A. (2016). The decoupling model of equanimity: theory, measurement, and test in a mindfulness intervention. Mindfulness, 7(5), 1214-1226. https://doi.org/10.1007/s12671-016-0564-2.

Kabat-Zinn, J. (1990). Full catastrophe living: the program of the stress reduction clinic at the University of Massachusetts Medical Center. Chicago.

Kabat-Zinn, J. (2003). Mindfulness-based interventions in context: past, present, and future. Clinical Psychology: Science and Practice, 10(2), 144-156. https://doi.org/10.1093/clipsy/bpg016.

Kane, M. J., Brown, L. H., McVay, J. C., Silvia, P. J., Myin-Germeys, I., $\&$ Kwapil, T. R. (2007). For whom the mind wanders, and when: an experience-sampling study of working memory and executive control in daily life. Psychological Science, 18(7), 614-621. https://doi. org/10.1111/j.1467-9280.2007.01948.x.

Kumari, V., Antonova, E., Wright, B., Hamid, A., Hernandez, E. M., Schmechtig, A., \& Ettinger, U. (2017). The mindful eye: smooth pursuit and saccadic eye movements in meditators and non-meditators. Consciousness and Cognition, 48, 66-75. https://doi.org/10. 1016/j.concog.2016.10.008.

Lee, K., \& Ashton, M. C. (2005). Psychopathy, Machiavellianism, and narcissism in the Five-Factor Model and the HEXACO model of personality structure. Personality and Individual Differences, 38(7), 1571-1582. https://doi.org/10.1016/j.paid.2004.09.016.

Lee, D. G., Kelly, K. R., \& Edwards, J. K. (2006). A closer look at the relationships among trait procrastination, neuroticism, and conscientiousness. Personality and Individual Differences, 40(1), 27-37. https://doi.org/10.1016/j.paid.2005.05.010.

Mantzios, M. (2018). Mindfulness breathing meditation [Audio File]. Available upon request from the author.

Marteau, T. M., \& Bekker, H. (1992). The development of a six-item shortform of the state scale of the Spielberger State-Trait Anxiety Inventory (STAI). British Journal of Clinical Psychology, 31(3), 301-306. https:// doi.org/10.1111/j.2044-8260.1992.tb00997.x.

Matiz, A., Crescentini, C., Fabbro, A., Budai, R., Bergamasco, M., \& Fabbro, F. (2019). Spontaneous eye movements during focusedattention mindfulness meditation. PloS one, 14(1), e0210862. https://doi.org/10.1371/journal.pone.0210862.

McCrae, R. R., \& Costa, P. T. (1987). Validation of the five-factor model of personality across instruments and observers. Journal of Personality and Social Psychology, 52(1), 81-90. https://doi.org/ 10.1037/0022-3514.52.1.81.

Mohan, A., Sharma, R., \& Bijlani, R. L. (2011). Effect of meditation on stress-induced changes in cognitive functions. The Journal of Alternative and Complementary Medicine, 17(3), 207-212. https:// doi.org/10.1089/acm.2010.0142.

Norris, C. J., Creem, D., Hendler, R., \& Kober, H. (2018). Brief mindfulness meditation improves attention in novices: evidence from ERPs and moderation by neuroticism. Frontiers in Human Neuroscience, 12, 315. https://doi.org/10.3389/fnhum.2018.00315.

Perfect, T. J., Wagstaff, G. F., Moore, D., Andrews, B., Cleveland, V., Newcombe, S., et al. (2008). How can we help witnesses to remember more? It's an (eyes) open and shut case. Law and Human Behavior, 32(4), 314-324. https://doi.org/10.1007/s10979-0079109-5.

Perfect, T. J., Andrade, J., \& Eagan, I. (2011). Eye closure reduces the cross-modal memory impairment caused by auditory distraction. 
Journal of Experimental Psychology: Learning, Memory, and Cognition, 37(4), 1008-1013. https://doi.org/10.1037/a0022930.

Reichle, E. D., Reineberg, A. E., \& Schooler, J. W. (2010). Eye movements during mindless reading. Psychological Science, 21(9), 1300-1310. https://doi.org/10.1177/0956797610378686.

Robison, M. K., Gath, K. I., \& Unsworth, N. (2017). The neurotic wandering mind: an individual differences investigation of neuroticism, mind-wandering, and executive control. The Quarterly Journal of Experimental Psychology, 70(4), 649-663. https://doi.org/10.1080/ 17470218.2016.1145706.

Schreiner, I., \& Malcolm, J. P. (2008). The benefits of mindfulness meditation: changes in emotional states of depression, anxiety, and stress. Behaviour Change, 25(3), 156-168. https://doi.org/10.1375/ bech.25.3.156.

Smilek, D., Carriere, J. S., \& Cheyne, J. A. (2010). Out of mind, out of sight: eye blinking as indicator and embodiment of mind wandering. Psychological Science, 21(6), 786-789. https://doi.org/10.1177/ 0956797610368063.

Spielberger, R., Gorsuch, R., \& Lushene, R. (1970). STAI manual for the State-Trait Anxiety Inventory 1970. Palo Alto: Consulting Psychologists.

Tanay, G., \& Bernstein, A. (2013). State Mindfulness Scale (SMS): development and initial validation. Psychological Assessment, 25(4), 1286-1299. https://doi.org/10.1037/a0034044.
Thompson, B. L., \& Waltz, J. (2007). Everyday mindfulness and mindfulness meditation: overlapping constructs or not? Personality and Individual Differences, 43(7), 1875-1885. https://doi.org/10.1016/j. paid.2007.06.017.

Tluczek, A., Henriques, J. B., \& Brown, R. L. (2009). Support for the reliability and validity of a six-item state anxiety scale derived from the State-Trait Anxiety Inventory. Journal of nursing measurement, 17(1), 19. https://doi.org/10.1891/1061-3749.17.1.19.

Wong, W. P., Camfield, D. A., Woods, W., Sarris, J., \& Pipingas, A. (2015). Spectral power and functional connectivity changes during mindfulness meditation with eyes open: a magnetoencephalography (MEG) study in long-term meditators. International Journal of Psychophysiology, 98(1), 95-111. https://doi.org/10.1016/j. ijpsycho.2015.07.006.

Zeidan, F., Johnson, S. K., Diamond, B. J., David, Z., \& Goolkasian, P. (2010). Mindfulness meditation improves cognition: evidence of brief mental training. Consciousness and Cognition, 19(2), 597605. https://doi.org/10.1016/j.concog.2010.03.014.

Publisher's Note Springer Nature remains neutral with regard to jurisdictional claims in published maps and institutional affiliations. 